\title{
Vitamin D supplementation and clinical outcomes in COVID-19: a systematic review and meta-analysis
}

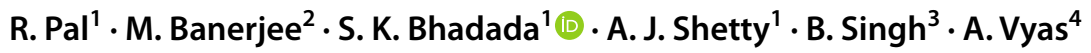

Received: 18 April 2021 / Accepted: 12 June 2021 / Published online: 24 June 2021

(C) Italian Society of Endocrinology (SIE) 2021

\begin{abstract}
Purpose To provide a precise summary and collate the hitherto available clinical evidence on the effect of vitamin D supplementation on clinical outcomes in COVID-19 patients.

Methods PubMed/MEDLINE, Scopus, and Web of Science databases were systematically searched using appropriate keywords till June 8, 2021, to identify observational studies and randomized controlled trials (RCTs) reporting adverse clinical outcomes (ICU admission and/or mortality) in COVID-19 patients receiving vitamin D supplementation vs. those not receiving the same. Both prior use and use of vitamin D after COVID-19 diagnosis were considered. Unadjusted/adjusted pooled odds ratio (OR) with 95\% confidence intervals (CI) were calculated (PROSPERO registration number CRD42021248488). Results We identified 13 studies (10 observational, 3 RCTs) pooling data retrieved from 2933 COVID-19 patients. Pooled analysis of unadjusted data showed that vitamin D use in COVID-19 was significantly associated with reduced ICU admission/mortality (OR $0.41,95 \%$ CI: $0.20,0.81, p=0.01, I^{2}=66 \%$, random-effects model). Similarly, on pooling adjusted risk estimates, vitamin D was also found to reduce the risk of adverse outcomes (pooled OR 0.27, 95\% CI: 0.08, 0.91, $p=0.03$, $I^{2}=80 \%$, random-effects model). Subgroup analysis showed that vitamin D supplementation was associated with improved clinical outcomes only in patients receiving the drug post-COVID-19 diagnosis and not in those who had received vitamin D before diagnosis.

Conclusions Vitamin D supplementation might be associated with improved clinical outcomes, especially when administered after the diagnosis of COVID-19. However, issues regarding the appropriate dose, duration, and mode of administration of vitamin D remain unanswered and need further research.
\end{abstract}

Keywords COVID-19 $\cdot$ Vitamin D $\cdot$ Mortality $\cdot$ ICU admission

R. Pal and M. Banerjee contributed equally.

S. K. Bhadada

bhadadask@rediffmail.com

1 Department of Endocrinology, Post Graduate Institute of Medical Education and Research, Chandigarh 160012, India

2 Department of Endocrinology, Institute of Postgraduate Medical Education and Research, Kolkata 700020, India

3 Government Medical College, Patiala 147001, India

4 Department of Medicine, Rabindranath Tagore Medical College, Udaipur 313001, India

\section{Introduction}

As the novel coronavirus disease (COVID-19) continues to rampage, the search for an effective treatment has hitherto been futile. Although anti-viral drugs efficacious against the Severe Acute Respiratory Syndrome Coronavirus 2 (SARS$\mathrm{CoV}-2$ ) remains to be earthed, vitamin $\mathrm{D}$ supplementation has been proposed to be an effective means for reducing the risk of COVID-19 infection and severity in some studies.

The use of vitamin D in COVID-19 has stemmed from the observations that apart from increasing the risk of acquiring SARS-CoV-2 [1, 2], hypovitaminosis D portends a poor prognosis for patients with COVID-19 [3]. Ample clinical evidence derived from observational and ecological studies suggest that vitamin D deficiency increases the risk for severe disease and mortality with COVID-19 [4-6]. Accordingly, vitamin D supplementation has been shown to 
improve clinical outcomes in COVID-19 [7-11]. Nevertheless, data is inconsistent with some observational studies and randomized controlled trials (RCTs) showing that vitamin D supplementation is not associated with improved clinical outcomes in COVID-19 [12-15]; on the contrary, a trend towards two-fold higher mortality was found in patients being routinely supplemented with vitamin $\mathrm{D}$ as compared to non-users [14].

Considering the heterogeneity in the available clinical evidence, the present systematic review and meta-analysis was undertaken to provide a precise summary and collate the effect of vitamin D supplementation on adverse clinical outcomes in COVID-19 patients.

\section{Methods}

This meta-analysis was conducted and reported according to the Preferred Reporting Items for Systematic reviews and Meta-analyses (PRISMA) statement [16]. The study protocol has been registered in PROSPERO (Registration number CRD42021248488) [17].

\section{Search strategy}

Two investigators (RP and MB) independently performed a systematic search of the literature across the PubMed/ MEDLINE, Scopus, and Web of Science databases from inception till June 8, 2021, using the following keywords interposed with appropriate Boolean operators: "COVID19" OR “SARS-CoV-2” AND “vitamin D supplementation." The language was restricted to English only. The references of relevant reviews and retrieved articles were also screened for potentially eligible articles. For missing data, the corresponding authors of the potentially eligible studies were contacted wherever possible.

\section{Eligibility and exclusion criteria}

Eligibility criteria were set as follows:

1. Given the scarcity of literature, both observational studies (prospective or retrospective, cohort or casecontrol design) as well as randomized controlled trials irrespective of study design (parallel/cross-over), study blinding (single-blind, double-blind, or open-label), and sample size would be included in the meta-analysis.

2. Studies should include patients with COVID-19, a proportion who must have been taking vitamin $\mathrm{D}$ prior to or after the diagnosis of COVID-19 irrespective of the dose, duration, or formulation of vitamin D used.
3. Studies should report clinical outcomes of COVID-19 patients in terms of the need for intensive care unit (ICU) admission or mortality or both.

4. The clinical outcomes should be reported as the rate of ICU admission/mortality (as the number of "events") in COVID-19 patients with vitamin D supplementation compared to those who did not receive vitamin D.

5. In addition, studies reporting the adjusted odds ratio (OR) or hazard ratio (HR) of ICU admission/mortality in COVID-19 patients with vitamin D supplementation compared to those who did not receive vitamin D supplementation were also included.

Exclusion criteria were set as follows:

1. Studies reporting clinical outcomes of COVID-19 patients other than ICU admission/mortality.

2. Clinical case series, study protocols, reviews, comments, editorials, letters to the editor.

3. Non-peer reviewed studies published as preprints.

4. Incompleteness in data.

\section{Data extraction}

Two investigators (RP and $\mathrm{MB}$ ) independently scanned titles and/or abstracts to exclude duplicate studies and studies that failed to meet the aforementioned eligibility criteria. Potentially eligible studies were full-text assessed. Any discrepancies between the aforementioned investigators were solved by discussion, consensus, or arbitration by a third senior investigator (SKB). Studies hence selected were reviewed, and the following data were extracted from full-text reports for further assessment: study characteristics, dose and duration of vitamin D supplementation, formulation and mode of vitamin D administration, the number of patients supplemented with vitamin $\mathrm{D}$, the number of COVID-19 patients with vitamin D supplementation who had experienced the reported clinical outcome as compared to those who did not receive vitamin D (i.e., the number of events in those supplemented with vitamin D vs. those not supplemented) and the adjusted OR/HR of ICU admission/mortality in those supplemented with vitamin D vs. those who did not receive vitamin D.

\section{Assessment of study quality}

The Newcastle-Ottawa Scale (NOS) was used to assess the quality and risk of bias of the included observational studies. The scale assesses three quality parameters, namely, selection, comparability, and outcome divided across eight specific items which slightly differ when scoring case-control and cohort studies [18]. The maximum score on NOS is 9 . Any score $\geq 7$ qualifies as high-quality with a low risk of 
bias, while a score $<5$ is categorized as low-quality with a high risk of inherent bias. Any score in between is rated as moderate-quality [19].

For RCTs, the risk of bias was assessed in the following domains using the corresponding Cochrane Collaboration's tool: random sequence generation, allocation concealment, blinding of participants and staff, blinding of outcome assessment, incomplete outcome data, and selective outcome reporting [20]. Each domain was rated as 'low', 'unclear', or 'high' risk of bias. Thus, for example, a study was rated as being of low risk of bias in the presence of adequate procedures in all the domains; on the contrary, an inadequate procedure in at least one domain rated a study as being of a high risk of bias. In any other case, a study was labeled as being of unclear risk of bias.

The assessment of study quality and risk of bias was independently conducted by two investigators (RP and AJS). Any discrepancy was solved by a discussion with a third senior investigator (SKB).

\section{Statistical analysis}

The difference in the rate of occurrence of ICU admission/ mortality (events) in COVID-19 patients supplemented with vitamin D vs. those not supplemented with vitamin D was calculated using the OR (unadjusted) with $95 \%$ confidence intervals (CI) after implementation of the Mantel-Haenszel (M-H) fixed-effects model. Adjusted estimates (OR or HR) from each study, wherever reported, were also pooled together using the generic inverse variance model with the fixed-effects formula. The OR and HR were pooled separately. Besides, wherever possible, we also performed a subgroup analysis of studies reporting the supplementation of vitamin D prior to the diagnosis of COVID-19 and those reporting the use of vitamin D post-COVID-19 diagnosis.

Statistical heterogeneity among studies was assessed using $I^{2}$ statistics. Heterogeneity was quantified as low, moderate, and high with upper limits of $25 \%, 50 \%$, and $75 \%$ for $I^{2}$, respectively [21]. In the present meta-analysis, significant heterogeneity was considered when the $I^{2}$ value was $\geq 50 \%$, with a $p$ value $<0.05$. Outcomes with significant heterogeneity were reanalyzed and reported using the random-effects model. A $p<0.05$ was considered to be statistically significant.

Statistical analysis was performed using the RevMan 5.4 software.

\section{Results}

After a scrupulous literature search and a meticulous study selection process, we included 13 studies in the present meta-analysis [7-15, 22-25], pooling data retrieved from
2933 patients with COVID-19 (Fig. 1). Of the 13 studies, 10 were observational studies [7-10, 12-14, 22-24], while 3 were RCTs [11, 15, 25]. Relevant studies where clinical outcomes were not reported in terms of ICU admission or mortality were excluded [26-28].

The primary characteristics of the included studies have been summarized in Table 1. Out of the 13 studies, 10 were hospital-based [7, 9-11, 13, 15, 22, 23, 25, 29]. The studies by Annweiler et al. [8] and Cangiano et al. [24] were nursing home-based studies, while that by Cereda et al. [14] had included three groups of COVID-19 cases; the first and second group consisted of Parkinson's disease patients and caregivers, respectively living in Lombardy, Italy while the third group was recruited from a referral hospital. The COVID-19 disease severity was infrequently reported across all the included studies.

Five studies catered to the use of vitamin $\mathrm{D}$ before the diagnosis of COVID-19 [9, 12, 14, 23, 24], while seven studies had reported the use of vitamin D after the diagnosis of COVID-19 [7, 10, 11, 13, 15, 22, 25]. Annweiler et al. [8] had considered the use of oral cholecalciferol in the week following the suspicion or diagnosis of COVID19 or during the previous month. Among the seven studies that catered to the use of vitamin $\mathrm{D}$ after the diagnosis of COVID-19, the exact time interval between onset of symptoms of COVID-19 and vitamin D supplementation was reported only by Murai et al. [15], where vitamin D was supplemented 10.3 days on average after the onset of COVID-19 symptoms.

The majority of the studies had used oral cholecalciferol, while Entrenas Castillo et al. [11] and Alcala-Diaz et al. [22] had used oral calcifediol. The dose of cholecalciferol used was highly variable across the included studies, ranging from 80,000 IU within a few hours of diagnosis of COVID19 to a maximum of $400,000 \mathrm{IU}$ supplemented as bolus oral cholecalciferol daily for 2 consecutive days (the second and third day of the in-hospital stay) $[7,10]$.

Mortality was the most commonly reported clinical outcome while ICU admission was reported in one study [11] and ICU transfer or all-cause in-hospital mortality was reported in another study [10]. The study by Ling et al. [9] included a primary cohort $(n=444)$ and a validation cohort $(n=542)$, both of which had been separately included in the present meta-analysis. Adjusted estimates of the clinical outcome (either OR or HR) were reported in eight studies [7-11, 14, 22, 23]. Among others, the most common covariates that were adjusted across all these six studies were age, sex, and presence or absence of comorbidities.

The study quality and the risk of bias for observational studies and RCTs have been depicted in Supplementary Table 1 and Supplementary Table 2, respectively. All the observational studies were of moderate or high quality (Supplementary Table 1). Of the three RCTs, the studies 


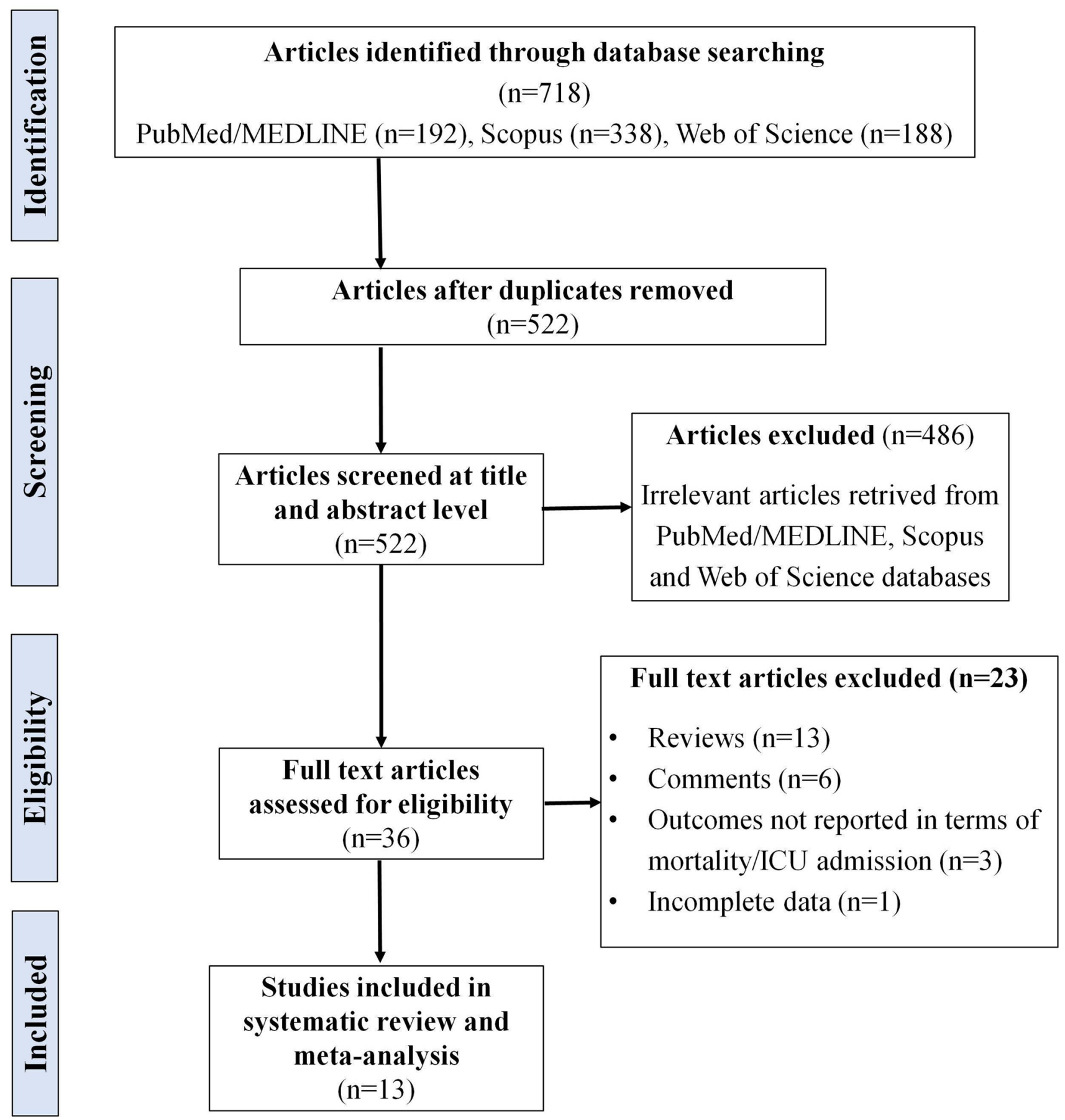

Fig. 1 Preferred reporting items for systematic reviews and meta-analyses (PRISMA) flowchart showing the study selection process

by Entrenas Castillo et al., and Lakkireddy et al., had high risk of bias, while that by Murai et al., had a low risk of bias (Supplementary Table 2). However, the RCT by Murai et al., had certain limitations. Of note, the baseline characteristics of the two groups (vitamin D group vs. placebo group) were not matched with the intervention group having a higher prevalence of diabetes, hypertension, and obesity. Besides, there were gender and racial differences between the two groups.

Nevertheless, none of the studies fulfilled the criteria required for the inclusion of clinical studies examining nutrient effects in systematic reviews and meta-analysis [30]. Of note, most of the included studies either did not mention or did not start from the same or similar basal nutrient status 


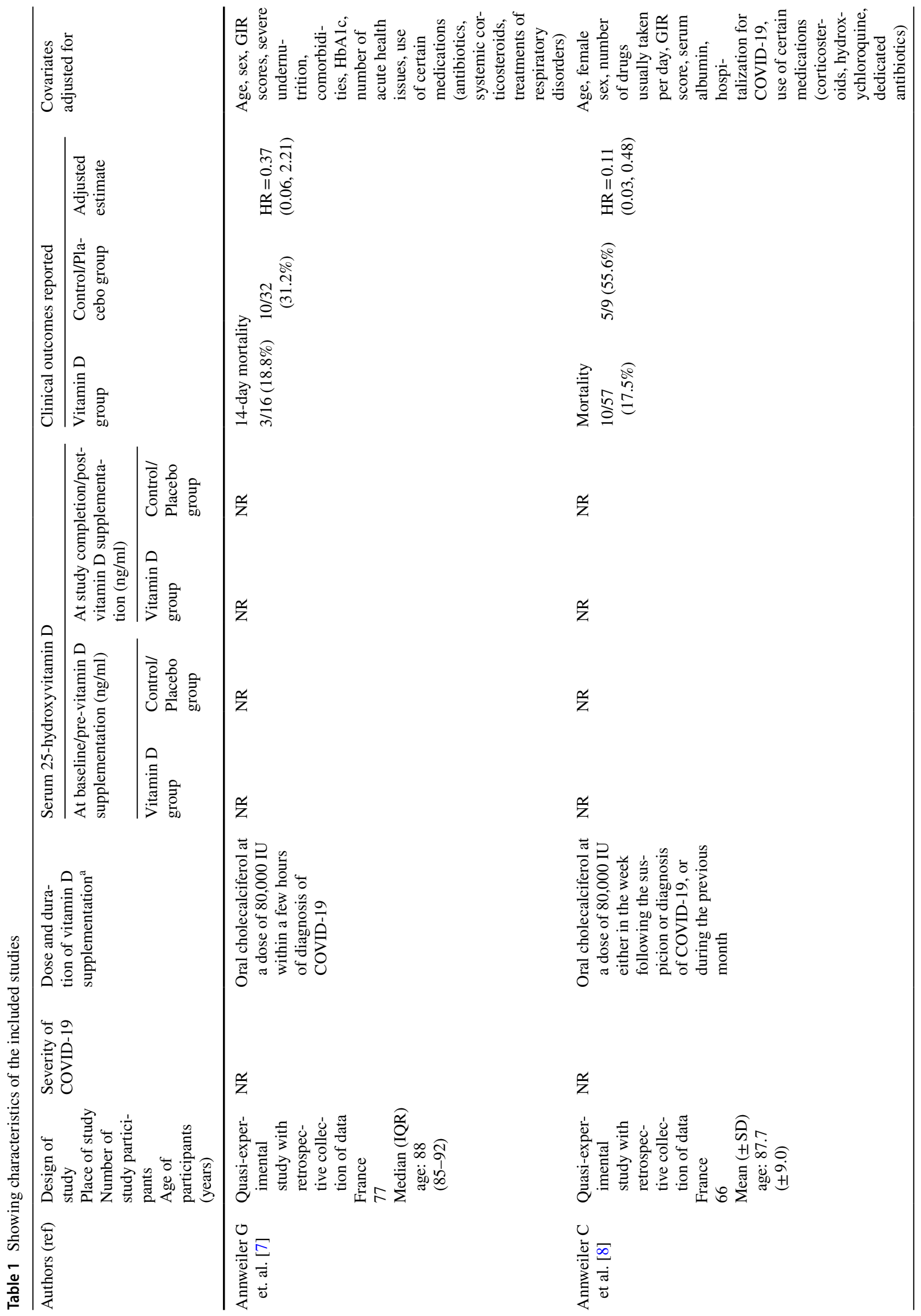




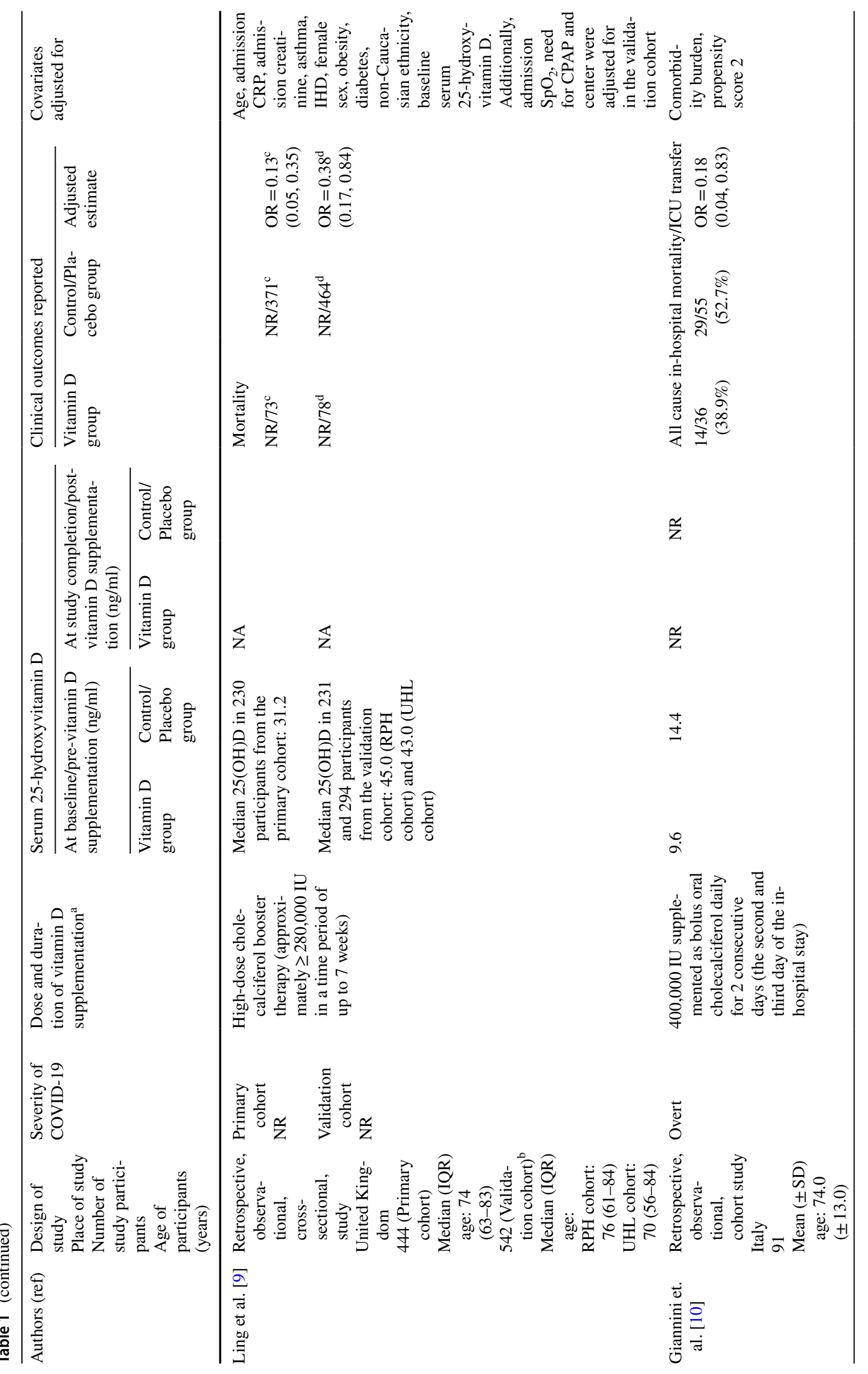




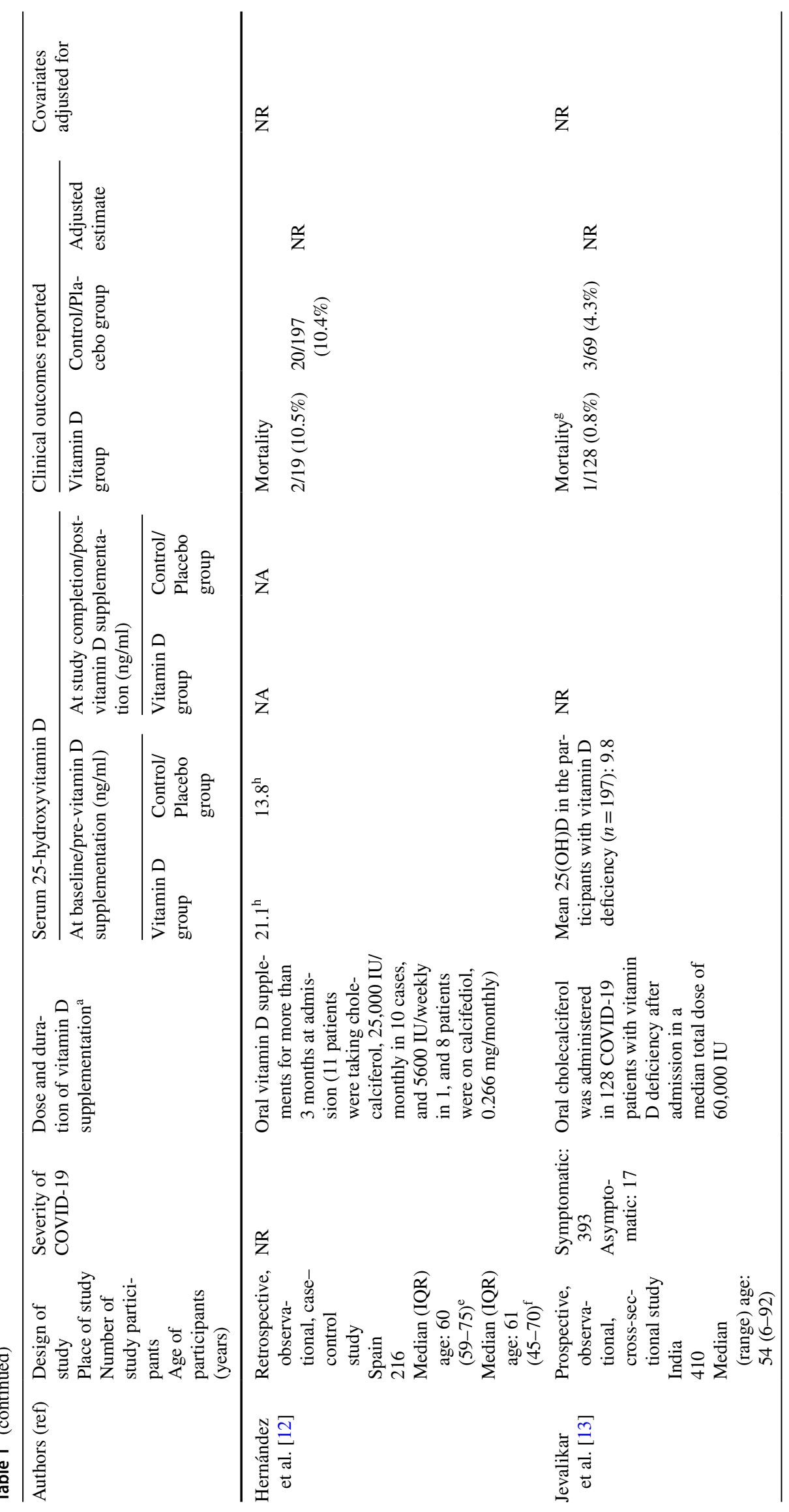




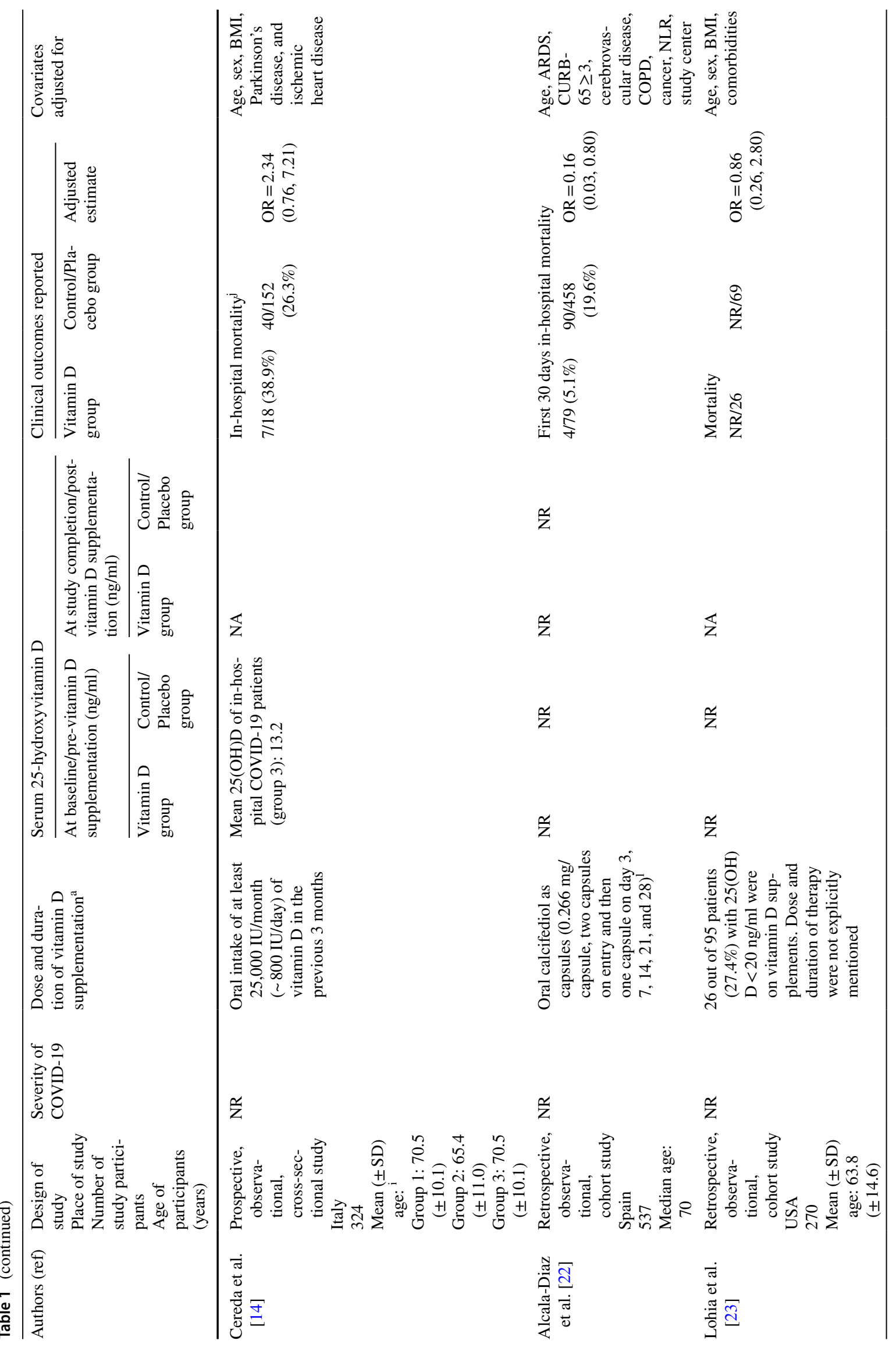




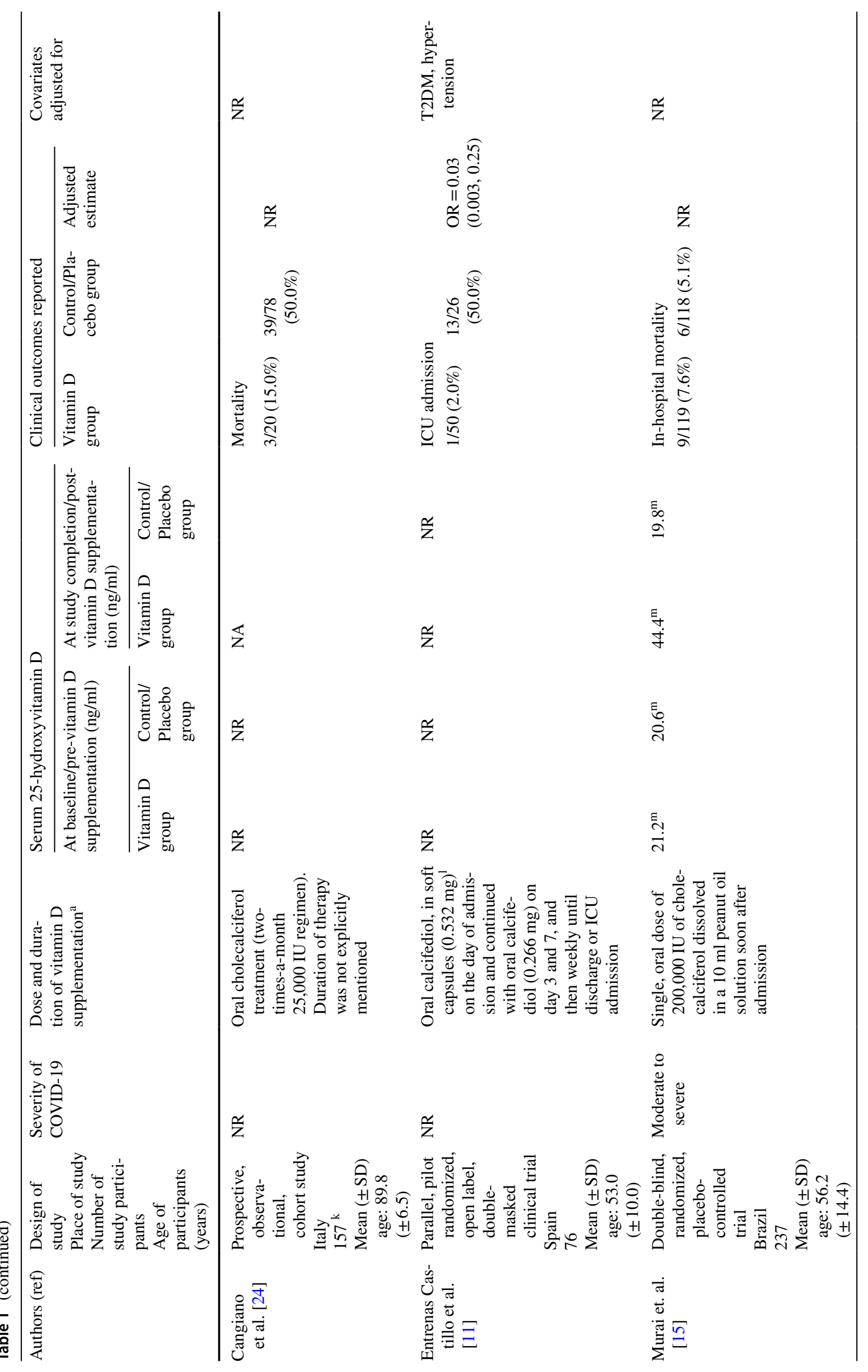




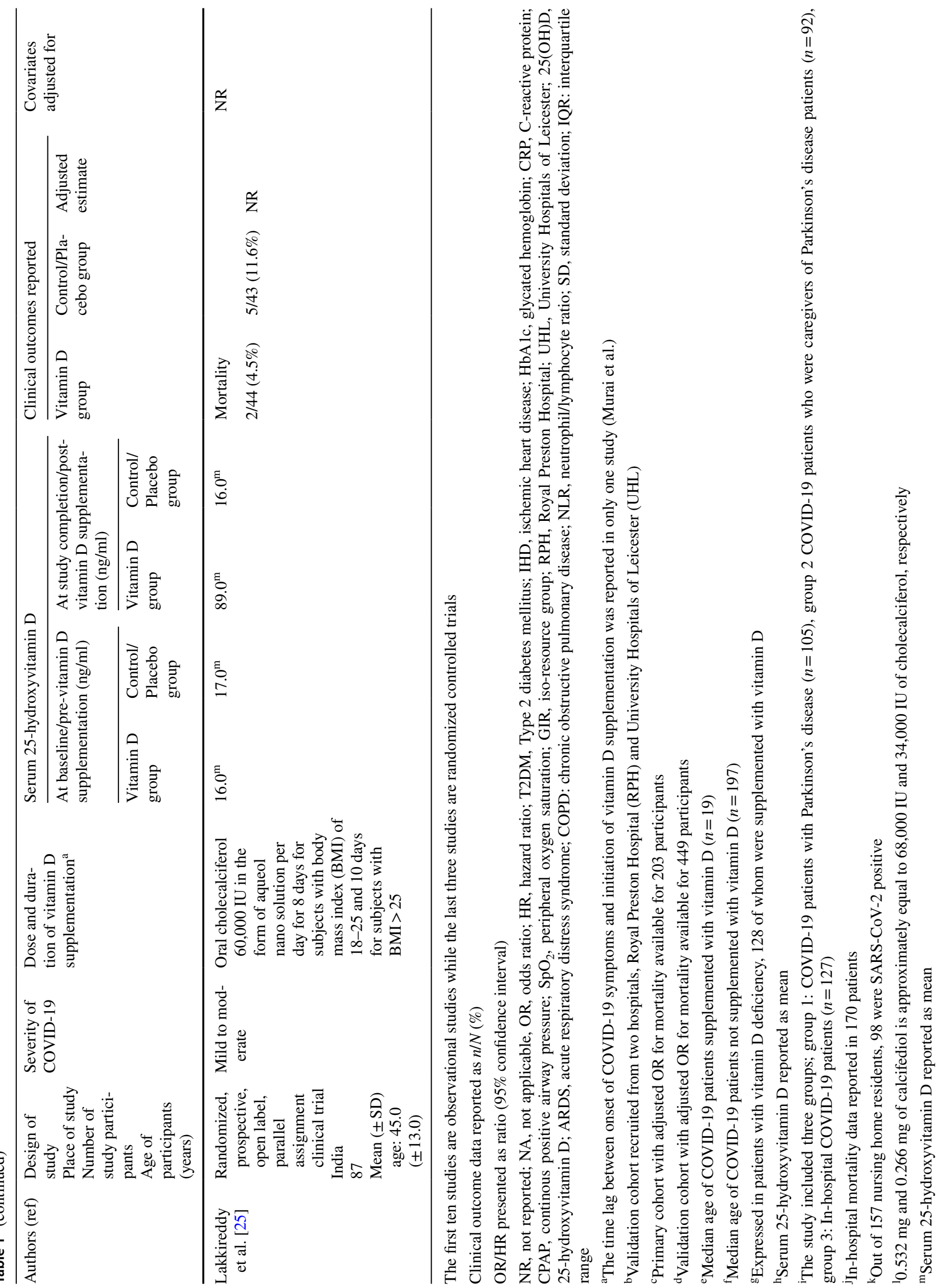


value (i.e., 25-hydroxyvitamin D), all the included studies did not use the same or closely similar doses, none of the studies reported about the conutrient status, and the periods of exposure to the increased intake of vitamin D were unequal amongst the included studies.

The results of the meta-analysis have been summarized under the following heads.

\section{Pooled analysis using the rate of occurrence of the reported clinical outcome (number of events) in COVID-19 patients receiving vs. those not receiving vitamin $D$}

The rate of occurrence of the reported clinical outcome (mortality or ICU admission or both) was explicitly mentioned in 11 studies [7, 8, 10-15, 22, 24, 25]. The pooled analysis of the data from the aforementioned studies showed that the use of vitamin D was associated with improved clinical outcomes (OR 0.41, 95\% CI: 0.20, 0.81, $p=0.01$, $I^{2}=66 \%$, random-effects model) (Fig. 2). A sensitivity analysis performed after excluding the RCT by Murai et al., showed similar results (OR 0.34, 95\% CI: 0.17, 0.69, $p=0.003, I^{2}=61 \%$, random-effects model) (Supplementary Fig. 1).

For subgroup analysis, the study by Annweiler et al. [8] was excluded as it catered to vitamin D supplementation prior to as well as after the diagnosis of COVID-19. Subgroup analysis showed that vitamin D supplementation was associated with improved clinical outcomes only in patients who had received vitamin $\mathrm{D}$ after the diagnosis of COVID-19 (OR 0.35, 95\% CI: 0.14, 0.85, $p=0.02, I^{2}=66 \%$, random-effects model, seven studies) but not in those who had received the same before COVID-19 diagnosis (OR $0.71,95 \%$ CI: $0.16,3.03, p=0.64, I^{2}=75 \%$, random-effects model, three studies) (Fig. 3). Sensitivity analysis performed after exclusion of the study by Murai et al., has been represented in Supplementary Fig. 2.

We performed another subgroup analysis based on the cumulative dose of vitamin $\mathrm{D}$ received as per the study protocol. A cumulative dose of cholecalciferol less than 200,000 IU was categorized as a low-cumulative dose, while a dose equal to or more than 200,000 IU was classified as a high-cumulative dose. Pooled data did not show any difference in outcome in the high-cumulative dose (OR 0.55, 95\% CI: $0.22,1.37, p=0.20, I^{2}=62 \%$, random-effects model, four studies) or low-cumulative dose subgroups (OR 0.34, 95\% CI: $0.10,1.18, p=0.09, I^{2}=74 \%$, random-effects model, five studies) (Supplementary Fig. 3). However, sensitivity analysis performed after exclusion of the RCT by Murai et al., showed that a high-cumulative dose of vitamin D was associated with improved clinical outcomes in COVID-19 (OR $0.38,95 \%$ CI: $0.20,0.72, p=0.003, I^{2}=4 \%$, random-effects model, three studies) (Supplementary Fig. 4).

\section{Pooled analysis using adjusted OR or HR of the reported clinical outcome in COVID-19 patients receiving vs. those not receiving vitamin D}

Adjusted OR of the reported clinical outcomes in COVID-19 patients supplemented with vitamin $\mathrm{D}$ vs. those not receiving vitamin D were reported in six studies [9-11, 14, 22, 23]. Pooled analysis showed that vitamin D use was significantly associated with improved clinical outcomes (pooled OR $0.31,95 \%$ CI: $0.12,0.78, p=0.01, I^{2}=74 \%$, random-effects model) (Fig. 4A). Subgroup analysis showed that vitamin D supplementation was associated with improved clinical outcomes only in patients receiving the drug after the diagnosis of COVID-19 (OR 0.12, 95\% CI: 0.04, 0.34, $p<0.0001$, $I^{2}=0 \%$, random-effects model, three studies) and not in those who had received vitamin $\mathrm{D}$ prior to the diagnosis (OR

\begin{tabular}{|c|c|c|c|c|c|}
\hline \multirow[b]{2}{*}{ Study or Subgroup } & \multicolumn{2}{|c|}{ Vitamin D } & \multicolumn{2}{|c|}{ Control/Placebo } & \multirow[b]{2}{*}{ Weight } \\
\hline & Events & Total & Events & Total & \\
\hline Jevalikar et al. (2021) & 1 & 128 & 3 & 69 & $5.6 \%$ \\
\hline Entrenas Castillo et al. (2020) & 1 & 50 & 13 & 26 & $6.1 \%$ \\
\hline Lakkireddy et al. (2021) & 2 & 44 & 5 & 43 & $7.8 \%$ \\
\hline Hernandez et al. (2020) & 2 & 19 & 20 & 197 & $8.5 \%$ \\
\hline Anmweiler C et al. (2020) & 10 & 57 & 5 & 9 & $8.8 \%$ \\
\hline Annweiler $G$ et al. (2020) & 3 & 16 & 10 & 32 & $8.9 \%$ \\
\hline Cangiano et al. (2020) & 3 & 20 & 39 & 78 & $9.6 \%$ \\
\hline Murai et al. (2021) & 9 & 119 & 5 & 118 & $10.6 \%$ \\
\hline Alcala-Diaz et al. (2021) & 4 & 79 & 90 & 458 & $11.0 \%$ \\
\hline Cereda et al. (2021) & 7 & 18 & 40 & 152 & $11.1 \%$ \\
\hline Giannini et al. (2021) & 14 & 36 & 29 & 55 & $12.0 \%$ \\
\hline Total $(95 \% \mathrm{CI})$ & & 586 & & 1237 & $100.0 \%$ \\
\hline Total events & 56 & & 259 & & \\
\hline $\begin{array}{l}\text { Heterogeneity: } \text { Tau }^{2}=0.85 ; \text { Chi } \\
\text { Test for overall effect: } Z=2.56\end{array}$ & $\begin{array}{l}=29.42, \\
=0.01)\end{array}$ & $d f=1$ & $=0.001)$ & $66 \%$ & \\
\hline
\end{tabular}

Odds Ratio Odds Ratio $-\mathrm{H}$, Random, $95 \% \mathrm{Cl}$ $0.17[0.02,1.70]$ $0.02[0.00,0.17]$ $0.36[0.07,1.98]$ $1.04[0.22,4.84]$ $0.17[0.04,0.75]$ $0.51[0.12,2.19]$ $0.18[0.05,0.65]$ $1.85[0.60,5.69]$ $0.22[0.08,0.61]$ $1.78[0.65,4.91]$ $0.57[0.24,1.34]$

$0.41[0.20,0.81]$

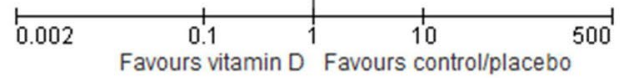

Fig. 2 Forest plot showing the effect (unadjusted) of vitamin D supplementation on clinical outcomes (intensive care unit admission and/or mortality) in patients with COVID-19 as compared to non-use of vitamin D 


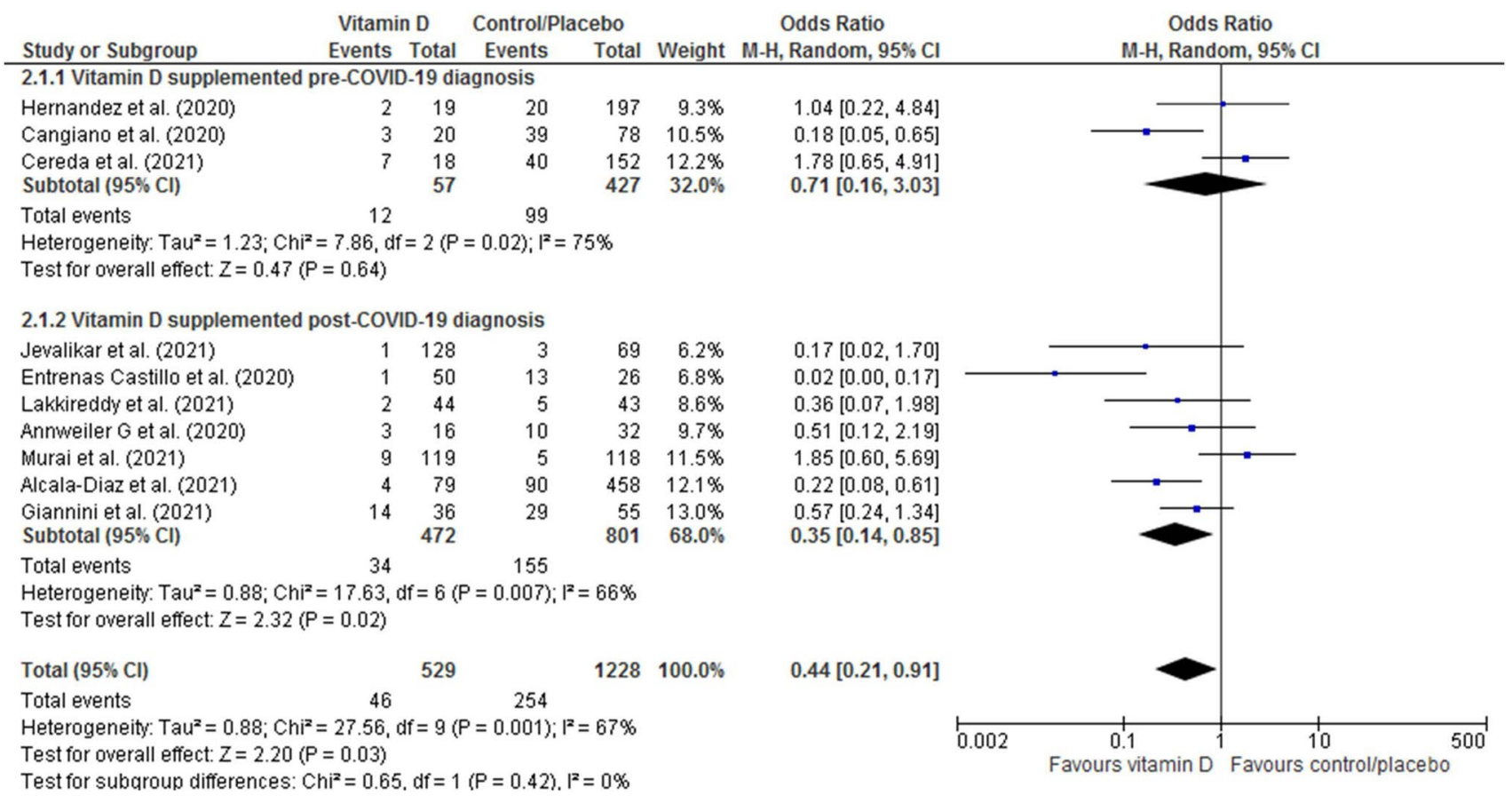

Fig. 3 Forest plot with subgroup analysis (based on the use of vitamin D pre- or post-COVID-19 diagnosis) showing the effect (unadcare unit admission and/or mortality) in patients with COVID-19 as compared to non-use of vitamin D

\begin{tabular}{|c|c|c|c|}
\hline Study or Subgroup & log[Odds Ratio] & SE & Weight \\
\hline Entrenas Castillo et al. (2020) & -3.5066 & 1.1748 & $9.0 \%$ \\
\hline Alcala-Diaz et al. (2021) & -1.8326 & 0.8541 & $12.2 \%$ \\
\hline Giannini et al. (2021) & -1.7148 & 0.7674 & $13.3 \%$ \\
\hline Lohia et al. (2021) & -0.1508 & 0.6103 & $15.2 \%$ \\
\hline Cereda et al. (2021) & 0.8502 & 0.5738 & $15.7 \%$ \\
\hline Ling et al. (2020) (Primary cohort) & -2.0402 & 0.4875 & $16.8 \%$ \\
\hline Ling et al. (2020) (validation cohort) & -0.9676 & 0.4104 & $17.7 \%$ \\
\hline Total $(95 \% \mathrm{Cl})$ & & & $100.0 \%$ \\
\hline \multicolumn{4}{|c|}{$\begin{array}{l}\text { Heterogeneity: } \text { Tau }^{2}=1.09 ; \mathrm{Chi}^{2}=23.25, \mathrm{df}=6(P=0.0007) ;\left.\right|^{2}=74 \% \\
\text { Test for overall effect: } Z=2.48(P=0.01)\end{array}$} \\
\hline
\end{tabular}

Odds Ratio

Random, $95 \% \mathrm{Cl}$

$0.03[0.00,0.30]$

$0.16[0.03,0.85]$

$0.18[0.04,0.81]$

$0.86[0.26,2.84]$

$2.34[0.76,7.21]$

$0.13[0.05,0.34]$

$0.38[0.17,0.85]$

$0.31[0.12,0.78]$

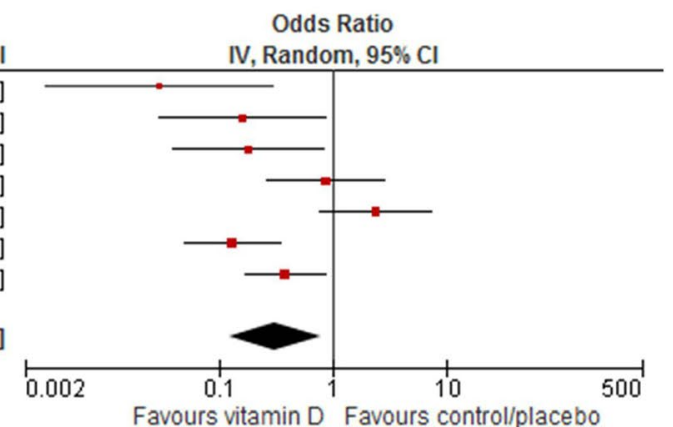

\section{B}

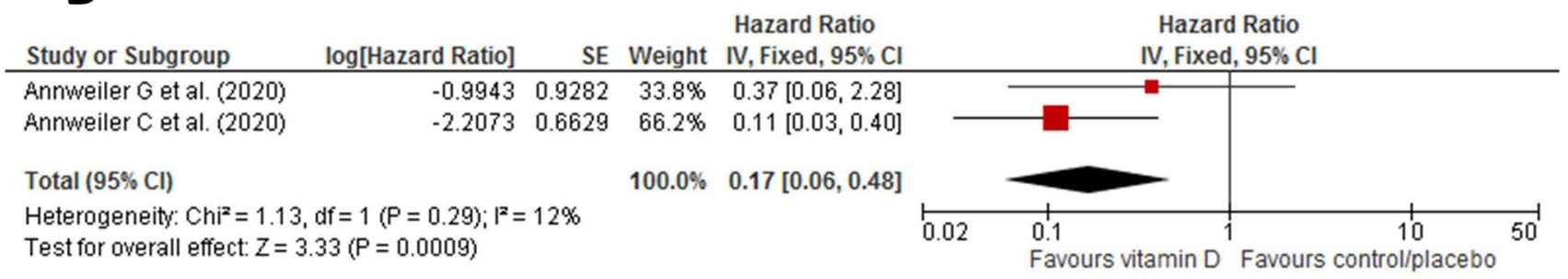

Fig. 4 Forest plots showing the effect (adjusted) of vitamin D supplementation on clinical outcomes (intensive care unit admission and/or mortality) in patients with COVID-19 as compared to non-use of vitamin D expressed either as pooled odds ratio (A) or pooled hazard ratio (B) 
$0.54,95 \%$ CI: $0.17,1.73, p=0.30, I^{2}=81 \%$, random-effects model, three studies) (Supplementary Fig. 5).

Similarly, covariate-adjusted HR was reported in only two studies [7, 8]. Pooled data also showed that vitamin D use was associated with improved clinical outcomes in COVID19 patients (pooled HR 0.17, 95\% CI: 0.06, 0.48, $p=0.0009$, $I^{2}=12 \%$, fixed-effects model) (Fig. 4B).

\section{Discussion}

The present systematic review and meta-analysis shows that supplementation with vitamin $\mathrm{D}$ is associated with improved clinical outcomes in COVID-19, especially when vitamin D is administered in patients after the diagnosis of COVID-19. Thus, vitamin D supplementation might be considered as a potential treatment adjunct in patients with COVID-19.

Multiple observational and ecological studies have shown that hypovitaminosis D is a risk factor for COVID-19 infection $[1,2,31,32]$. In a retrospective, observational study that had included 191,779 COVID-19 patients in whom a serum 25-hydroxyvitamin D level over the preceding 12 months was available, there was an independent association between higher SARS-CoV-2 positivity rates and lower circulating 25-hydroxyvitamin D levels. Although 25-hydroxyvitamin D levels appeared to play a role for all race/ethnicities, patients from predominately black non-Hispanic zip codes had higher SARS-CoV-2 positivity than those from predominately white non-Hispanic zip codes at every 25 -hydroxyvitamin $D$ level, suggesting a greater impact of ethnicity on the risk of developing COVID-19 [31].

As is the case with COVID-19 infection, there is ample data to suggest that vitamin D deficiency is associated with COVID-19 severity and mortality [4-6, 33]. Although contradictory literature exists wherein the relationship between vitamin D and COVID-19 has been negated [13], several systematic reviews and meta-analyses, albeit based on observational studies, suggest a consistent negative association between serum 25-hydroxyvitamin D levels and COVID-19 disease severity [3, 34-37].

Several mechanisms have been hypothesized to explain the association between vitamin D and COVID-19 [38-40]. One is through mounting a defense against the virus, partly through induction of cathelicidin (LL-37) and defensins. LL-37 acts at several steps in viral infection and is effective against both enveloped and non-enveloped viruses [41]. Besides, higher levels of LL-37 in serum correspond to lower expression of interleukin-17 (IL-17). Hitherto, data suggest that IL-17 is involved in the pathology of COVID19, including the risk of thrombosis [42] and acute respiratory distress syndrome (ARDS) [43]. Thus, upregulation of IL-17 might explain the link between hypovitaminosis D and COVID-19 severity and acute complications.
A second mechanism linking vitamin D and COVID-19 is the regulation of production of cytokines. Vitamin D promotes upregulation of anti-inflammatory cytokines such as IL-10, and downregulation of proinflammatory cytokines such as IL-1, IL-6 and tumor-necrosis factor-alpha. Such a shift from a proinflammatory to an anti-inflammatory state can reduce risk of the cytokine storm in COVID-19 [44].

A third mechanism is modulation of the renin-angiotensin-aldosterone system (RAAS) and angiotensin-converting enzyme 2 (ACE2). Vitamin D induces the ACE2/Ang (1-7) axis activity and inhibits renin and the ACE/Ang II/AT1R axis, thereby increasing the expression and concentration of ACE2 and Ang (1-7). ACE2/Ang (1-7) system plays an important anti-inflammatory and antioxidant role in protecting the lung against ARDS; indeed, ACE2 has been protective against lethal avian influenza A H5N1 infection [45, 46]. Thus, upregulation of the ACE2/Ang (1-7) system would have a potential protective role against acute lung injury and ARDS [40, 47].

Thus, supplementation of vitamin D in patients with COVID-19 might be expected to be rewarding. Expectedly, multiple observational and experimental studies have shown that vitamin D supplementation, either before or after the diagnosis of COVID-19, is associated with improved clinical outcomes in terms of ICU admission and/or mortality [7-11]. Nevertheless, contradictory data also exist wherein vitamin D supplementation has been shown not to be associated with improved clinical outcomes [13-15, 29]. However, it should be noted that most of these studies did not report adjusted risk estimates for clinical outcomes after adjustment for potential confounding factors [13, 15, 29].

The present meta-analysis shows that vitamin D supplementation is associated with improved clinical outcomes in patients with COVID-19. Besides, subgroup analysis showed that the patients supplemented with vitamin D after the diagnosis of COVID-19 were more likely to benefit rather than those supplemented with the drug prior to the diagnosis. Two studies catering to the use of vitamin D after the diagnosis of COVID-19 that were included in the subgroup analysis had used cholecalciferol/calcifediol at a cumulatively high dose $[10,11]$. Thus, it might be prudent to consider the use of vitamin D as a potential therapeutic adjunct in COVID-19 patients, especially those with a moderate-to-severe disease requiring hospitalization. However, the appropriate dose and duration of vitamin D supplementation remain yet to be explored.

The present meta-analysis happens to be the most comprehensive pooled data regarding vitamin D supplementation on clinical outcomes in COVID-19. A somewhat similar meta-analysis published earlier in 2021 found no significant benefit of vitamin D supplementation on mortality in COVID-19; however, the meta-analysis had included only three studies. Besides, the authors had presented 
only unadjusted risk estimates, thereby failing to consider potential confounding factors [48]. On the other hand, we have pooled data from 13 studies and have provided adjusted apart from unadjusted risk estimates, to make the results more robust and generalizable.

We humbly acknowledge the limitations of the metaanalysis. First, adjusted estimates were not reported in some studies, hence, they could not be included in the adjusted pooled analysis. In addition, the covariates reported across all the included studies were not uniform, and the OR/ HR derived from various studies was adjusted for different covariates. Second, most of the studies have considered the use of vitamin D irrespective of the baseline serum 25-hydroxyvitamin D levels of the patients; therefore, it is difficult to opine if the response to vitamin $\mathrm{D}$ supplementation would have been different in those with and without hypovitaminosis D. Data on baseline 25-hydroxyvitamin D levels were infrequently reported across all the studies, hence, a subgroup analysis based on the baseline vitamin D status was not feasible.

Third, the time lag between the onset of COVID-19 symptoms and vitamin D supplementation was sparsely reported, hence, subgroup analysis taking into consideration this time lag could not be performed. Notably, vitamin D was supplemented as late as 10.3 days (mean) after symptom onset, which might have circumvented the beneficial effects of vitamin D had it been supplemented early in the course of the disease [15, 49]. Fourth, data on COVID-19 severity were infrequently and inconsistently reported across all the studies, hence, we could not perform a subgroup analysis based on the underlying disease severity. Besides, considering the abundant data showing intersex differences in COVID-19 severity and mortality, a separate subgroup analysis depicting the effect of vitamin D supplementation on men and women would have been worthwhile. However, the same could not be performed due to the lack of such data.

Fifth, except for the RCTs by Murai et al., and Lakkireddy et al., none of the studies mention the degree of rise in serum 25-hydroxyvitamin D levels post-vitamin D supplementation, hence, we can only speculate if adequate vitamin D levels had been achieved to exert immunomodulatory effects. Although the appropriate level of 25-hydroxyvitamin D levels required for its immunomodulatory action is not explicitly known [26], it has been reported that 25-hydroxyvitamin D levels $>30 \mathrm{ng} / \mathrm{ml}$ are associated with a significant decrease in the SARS-CoV-2 infection severity and mortality [50].

Lastly, in the absence of data on serum 25-hydroxyvitamin D levels, it remains uncertain if a single high-dose bolus or daily low dose of vitamin $\mathrm{D}$ would be more effective. In general, a single high-dose bolus of vitamin $\mathrm{D}$, as used in the studies by Murai et al., and Giannini et al., is generally sufficient to achieve adequate 25 -hydroxyvitamin D levels in the first 3-5 days following administration [51,
52]; however, high-dose bolus replacement induces longterm expression of the catabolic enzyme 24-hydroxylase and fibroblast growth factor 23 (FGF23). Increased expression of 24-hydroxylase leads to diversion of 25-hydroxyvitamin D to inactive 24,25-dihydroxyvitamin D while FGF23 leads to inactivation of the enzyme renal $1 \alpha$-hydroxylase that reduces the generation of the active metabolite, calcitriol [53]. On the contrary, a daily dose of vitamin D has more lasting effects in increasing 25-hydroxyvitamin D levels [54]. In this regard, maintenance doses of vitamin D administered after a single bolus dose can be expected to maintain sufficient vitamin $\mathrm{D}$ levels for a longer duration of time.

In conclusion, the present systematic review and metaanalysis suggests that vitamin D supplementation might be associated with improved clinical outcomes in terms of ICU admission and/or mortality, especially in those with moderate-to-severe COVID-19 requiring hospitalization. However, issues regarding the appropriate dose, duration, and mode of administration of vitamin D remain unanswered and provide avenues for further research.

Supplementary Information The online version contains supplementary material available at https://doi.org/10.1007/s40618-021-01614-4.

Author contributions RP and MB are the co-primary authors, had performed literature search, data extraction and statistical analysis. SKB is the corresponding author, had conceptualized, supervised and edited the manuscript. AJS had helped in risk of bias assessment. BS and AV had helped in statistical analysis and had edited the manuscript. All the authors approved the final version of the manuscript.

Funding None.

Availability of data and material Not applicable.

\section{Declarations}

Conflicts of interest None to declare.

Ethical approval This article does not contain any studies with human participants or animals performed by any of the authors.

Informed consent No informed consent.

\section{References}

1. Meltzer DO, Best TJ, Zhang $\mathrm{H}$ et al (2020) Association of vitamin D status and other clinical characteristics with COVID-19 test results. JAMA Netw Open 3:e2019722. https://doi.org/10.1001/ jamanetworkopen.2020.19722

2. Pal R, Ram S, Zohmangaihi D et al (2020) High prevalence of hypocalcemia in non-severe COVID-19 patients: a retrospective case-control study. Front Med 7:590805. https://doi.org/10.3389/ fmed.2020.590805 
3. Munshi R, Hussein MH, Toraih EA et al (2021) Vitamin D insufficiency as a potential culprit in critical COVID-19 patients. J Med Virol 93:733-740. https://doi.org/10.1002/jmv.26360

4. Mariani J, Giménez VMM, Bergam I et al (2020) Association between vitamin D deficiency and COVID-19 incidence, complications, and mortality in 46 countries: an ecological study. Health Secur. https://doi.org/10.1089/hs.2020.0137

5. Radujkovic A, Hippchen T, Tiwari-Heckler S et al (2020) Vitamin D deficiency and outcome of COVID-19 patients. Nutrients. https://doi.org/10.3390/nu12092757

6. Baktash V, Hosack T, Patel N et al (2020) Vitamin D status and outcomes for hospitalised older patients with COVID-19. Postgrad Med J. https://doi.org/10.1136/postgradmedj-2020-138712

7. Annweiler G, Corvaisier M, Gautier J et al (2020) Vitamin D supplementation associated to better survival in hospitalized frail elderly COVID-19 patients: the GERIA-COVID quasiexperimental study. Nutrients 12:3377. https://doi.org/10.3390/ nu12113377

8. Annweiler C, Hanotte B, Grandin de l'Eprevier C et al (2020) Vitamin D and survival in COVID-19 patients: a quasi-experimental study. J Steroid Biochem Mol Biol 204:105771. https:// doi.org/10.1016/j.jsbmb.2020.105771

9. Ling SF, Broad E, Murphy R et al (2020) High-dose cholecalciferol booster therapy is associated with a reduced risk of mortality in patients with COVID-19: a cross-sectional multi-centre observational study. Nutrients 12:3799. https://doi.org/10.3390/nu121 23799

10. Giannini S, Passeri G, Tripepi G et al (2021) Effectiveness of in-hospital cholecalciferol use on clinical outcomes in comorbid COVID-19 patients: a hypothesis-generating study. Nutrients 13:219. https://doi.org/10.3390/nu13010219

11. Entrenas Castillo M, Entrenas Costa LM, Vaquero Barrios JM et al (2020) Effect of calcifediol treatment and best available therapy versus best available therapy on intensive care unit admission and mortality among patients hospitalized for COVID-19: a pilot randomized clinical study. J Steroid Biochem Mol Biol 203:105751. https://doi.org/10.1016/j.jsbmb.2020.105751

12. Hernández JL, Nan D, Fernandez-Ayala M et al (2020) Vitamin D status in hospitalized patients with SARS-CoV-2 infection. J Clin Endocrinol Metab. https://doi.org/10.1210/clinem/dgaa733

13. Jevalikar G, Mithal A, Singh A et al (2021) Lack of association of baseline 25-hydroxyvitamin D levels with disease severity and mortality in Indian patients hospitalized for COVID-19. Sci Rep 11:6258. https://doi.org/10.1038/s41598-021-85809-y

14. Cereda E, Bogliolo L, Lobascio F et al (2021) Vitamin D supplementation and outcomes in coronavirus disease 2019 (COVID19) patients from the outbreak area of Lombardy. Italy Nutrition 82:111055. https://doi.org/10.1016/j.nut.2020.111055

15. Murai IH, Fernandes AL, Sales LP et al (2021) Effect of a single high dose of vitamin $\mathrm{D}_{3}$ on hospital length of stay in patients with moderate to severe COVID-19: a randomized clinical trial. JAMA 325:1053. https://doi.org/10.1001/jama.2020.26848

16. Liberati A, Altman DG, Tetzlaff J et al (2009) The PRISMA statement for reporting systematic reviews and meta-analyses of studies that evaluate healthcare interventions: explanation and elaboration. BMJ 339:b2700-b2700. https://doi.org/10.1136/bmj.b2700

17. Pal R, Bhadada SK (2021) Vitamin D supplementation and adverse clinical outcomes in COVID-19: a systematic review and meta-analysis. PROSPERO 2021 CRD42021248488. https:// www.crd.york.ac.uk/PROSPERO/display_record.php?RecordID= 248488

18. Wells G, Shea B, O'Connell D et al (2013) The Newcastle-Ottawa Scale (NOS) for assessing the quality of nonrandomised studies in meta-analyses. http://www.ohri.ca/programs/clinical_epidemiolo gy/oxford.asp
19. Luchini C, Stubbs B, Solmi M, Veronese N (2017) Assessing the quality of studies in meta-analyses: advantages and limitations of the Newcastle Ottawa Scale. World J Meta-Anal 5:80. https://doi. org/10.13105/wjma.v5.i4.80

20. Higgins J, Sterne J, Savovic J et al (2016) A revised tool for assessing risk of bias in randomized trials. Cochrane Database Syst Rev 10:29-31

21. Higgins JPT (2003) Measuring inconsistency in meta-analyses. BMJ 327:557-560. https://doi.org/10.1136/bmj.327.7414.557

22. Alcala-Diaz JF, Limia-Perez L, Gomez-Huelgas R et al (2021) Calcifediol treatment and hospital mortality due to COVID-19: a cohort study. Nutrients 13:1760. https://doi.org/10.3390/nu130 61760

23. Lohia P, Nguyen P, Patel N, Kapur S (2021) Exploring the link between vitamin $\mathrm{D}$ and clinical outcomes in COVID-19. Am J Physiol-Endocrinol Metab 320:E520-E526. https://doi.org/10. 1152/ajpendo.00517.2020

24. Cangiano B, Fatti LM, Danesi L et al (2020) Mortality in an Italian nursing home during COVID-19 pandemic: correlation with gender, age, ADL, vitamin D supplementation, and limitations of the diagnostic tests. Aging 12:24522-24534. https://doi.org/10. 18632/aging.202307

25. Lakkireddy M, Gadiga SG, Malathi RD et al (2021) Impact of daily high dose oral vitamin $\mathrm{D}$ therapy on the inflammatory markers in patients with COVID 19 disease. Sci Rep 11:10641. https:// doi.org/10.1038/s41598-021-90189-4

26. Rastogi A, Bhansali A, Khare N et al (2020) Short term, high-dose vitamin D supplementation for COVID-19 disease: a randomised, placebo-controlled, study (SHADE study). Postgrad Med J postgrad. https://doi.org/10.1136/postgradmedj-2020-139065

27. Sánchez-Zuno GA, González-Estevez G, Matuz-Flores MG et al (2021) Vitamin D levels in COVID-19 outpatients from Western Mexico: clinical correlation and effect of its supplementation. J Clin Med 10:2378. https://doi.org/10.3390/jcm10112378

28. Tan CW, Ho LP, Kalimuddin S et al (2020) Cohort study to evaluate the effect of vitamin D, magnesium, and vitamin B12 in combination on progression to severe outcomes in older patients with coronavirus (COVID-19). Nutrition 79-80:111017. https://doi. org/10.1016/j.nut.2020.111017

29. Hernández JL, Nan D, Fernandez-Ayala M et al (2021) Vitamin D status in hospitalized patients with SARS-CoV-2 infection. J Clin Endocrinol Metab 106:e1343-e1353. https://doi.org/10.1210/ clinem/dgaa733

30. Heaney RP (2014) Guidelines for optimizing design and analysis of clinical studies of nutrient effects. Nutr Rev 72:48-54. https:// doi.org/10.1111/nure. 12090

31. Kaufman HW, Niles JK, Kroll MH et al (2020) SARS-CoV-2 positivity rates associated with circulating 25-hydroxyvitamin D levels. PLoS ONE 15:e0239252. https://doi.org/10.1371/journal. pone. 0239252

32. Shah Alam M, Czajkowsky DM, Aminul Islam Md, Ataur Rahman Md (2021) The role of vitamin D in reducing SARS-CoV-2 infection: an update. Int Immunopharmacol 97:107686. https:// doi.org/10.1016/j.intimp.2021.107686

33. Charoenngam N, Shirvani A, Reddy N et al (2021) Association of vitamin D status with hospital morbidity and mortality in adult hospitalized patients with COVID-19. Endocr Pract 27:271-278. https://doi.org/10.1016/j.eprac.2021.02.013

34. Akbar MR, Wibowo A, Pranata R, Setiabudiawan B (2021) Low Serum 25-hydroxyvitamin D (Vitamin D) level is associated with susceptibility to COVID-19, severity, and mortality: a systematic review and meta-analysis. Front Nutr 8:660420. https://doi.org/ 10.3389/fnut.2021.660420

35. Teshome A, Adane A, Girma B, Mekonnen ZA (2021) The impact of vitamin D level on COVID-19 infection: systematic review and 
meta-analysis. Front Public Health 9:624559. https://doi.org/10. 3389/fpubh.2021.624559

36. Pereira M, Dantas Damascena A, Galvão Azevedo LM et al (2020) Vitamin D deficiency aggravates COVID-19: systematic review and meta-analysis. Crit Rev Food Sci Nutr. https://doi.org/10. 1080/10408398.2020.1841090

37. Bassatne A, Basbous M, Chakhtoura M et al (2021) The link between COVID-19 and VItamin D (VIVID): a systematic review and meta-analysis. Metabolism. https://doi.org/10.1016/j.metabol. 2021.154753

38. Mercola J, Grant WB, Wagner CL (2020) Evidence regarding vitamin D and Risk of COVID-19 and its severity. Nutrients 12:3361. https://doi.org/10.3390/nu12113361

39. Grant WB, Lahore H, McDonnell SL et al (2020) Evidence that vitamin D Supplementation could reduce risk of influenza and COVID-19 infections and deaths. Nutrients 12:988. https://doi. org/10.3390/nu12040988

40. Grant WB, Lahore H, Rockwell MS (2020) The benefits of vitamin D supplementation for athletes: better performance and reduced risk of COVID-19. Nutrients 12:3741. https://doi.org/ $10.3390 /$ nu 12123741

41. Brice DC, Diamond G (2020) Antiviral activities of human host defense peptides. Curr Med Chem 27:1420-1443. https://doi.org/ 10.2174/0929867326666190805151654

42. Raucci F, Mansour AA, Casillo GM et al (2020) Interleukin-17A (IL-17A), a key molecule of innate and adaptive immunity, and its potential involvement in COVID-19-related thrombotic and vascular mechanisms. Autoimmun Rev 19:102572. https://doi.org/ 10.1016/j.autrev.2020.102572

43. Pacha O, Sallman MA, Evans SE (2020) COVID-19: a case for inhibiting IL-17? Nat Rev Immunol 20:345-346. https://doi.org/ 10.1038/s41577-020-0328-Z

44. Bilezikian JP, Bikle D, Hewison M et al (2020) Mechanisms in endocrinology: vitamin D and COVID-19. Eur J Endocrinol 183:R133-R147. https://doi.org/10.1530/EJE-20-0665

45. Zou Z, Yan Y, Shu Y et al (2014) Angiotensin-converting enzyme 2 protects from lethal avian influenza A H5N1 infections. Nat Commun. https://doi.org/10.1038/ncomms4594

46. Pal R, Bhansali A (2020) COVID-19, diabetes mellitus and ACE2: the conundrum. Diabetes Res Clin Pract 162:108132. https://doi. org/10.1016/j.diabres.2020.108132
47. Malek Mahdavi A (2020) A brief review of interplay between vitamin $\mathrm{D}$ and angiotensin-converting enzyme 2: implications for a potential treatment for COVID-19. Rev Med Virol. https://doi. org/10.1002/rmv.2119

48. Shah K, Saxena D, Mavalankar D (2021) Vitamin D supplementation, COVID-19 and disease severity: a meta-analysis. QJM Int J Med. https://doi.org/10.1093/qjmed/hcab009

49. Annweiler C, Mercat A, Souberbielle J-C (2021) Learning from previous methodological pitfalls to propose well-designed trials on vitamin D in COVID-19. J Steroid Biochem Mol Biol 211:105901. https://doi.org/10.1016/j.jsbmb.2021.105901

50. Maghbooli Z, Sahraian MA, Ebrahimi M et al (2020) Vitamin D sufficiency, a serum 25-hydroxyvitamin D at least $30 \mathrm{ng} /$ $\mathrm{mL}$ reduced risk for adverse clinical outcomes in patients with COVID-19 infection. PLoS ONE 15:e0239799. https://doi.org/ 10.1371/journal.pone.0239799

51. Kearns MD, Binongo JNG, Watson D et al (2015) The effect of a single, large bolus of vitamin D in healthy adults over the winter and following year: a randomized, double-blind, placebocontrolled trial. Eur J Clin Nutr 69:193-197. https://doi.org/10. 1038/ejen.2014.209

52. Barker T, May HT, Doty JR et al (2021) Vitamin D supplementation protects against reductions in plasma 25-hydroxyvitamin D induced by open-heart surgery: Assess-d trial. Physiol Rep. https://doi.org/10.14814/phy2.14747

53. Griffin G, Hewison M, Hopkin J et al (2021) Perspective: Vitamin D supplementation prevents rickets and acute respiratory infections when given as daily maintenance but not as intermittent bolus: implications for COVID-19. Clin Med 21:e144-e149. https://doi.org/10.7861/clinmed.2021-0035

54. Ketha H, Thacher TD, Oberhelman SS et al (2018) Comparison of the effect of daily versus bolus dose maternal vitamin D3 supplementation on the 24,25-dihydroxyvitamin D3 to 25-hydroxyvitamin D3 ratio. Bone 110:321-325. https://doi.org/10.1016/j. bone.2018.02.024

Publisher's Note Springer Nature remains neutral with regard to jurisdictional claims in published maps and institutional affiliations. 